\title{
Correspondence
}

\section{A Unified Approach to the Change of Resolution: Space and Gray-Level}

\author{
SHMUEL PELEG, MICHAEL WERMAN, AND HILLEL ROM
}

\begin{abstract}
Multiple resolution analysis of images is a current trend in computer vision. In most cases, only spatial resolution has been considered. However, image resolution has an additional aspect: gray level, or color, resolution. Color resolution has traditionally been considered in the area of computer graphics.

By defining a suitable measure for the comparison of images, changes in resolution can be treated with the same tools as changes in color resolution. A gray tone image, for example, can be compared to a halftone image having only two colors (black and white), but of higher spatial resolution.

An important application can be in pyramids, one of the most commonly used multiple (spatial) resolution schemes, where this approach provides a tool to change the color resolution as well. Increasing color resolution while reducing spatial resolution to retain more image details and prevent aliasing is an example of the possibility to find optimal combinations of resolution reduction, spatial and color, to best fit an application.
\end{abstract}

Index Terms-Computer graphics, computer vision, image digitization, image resolution.

\section{INTRODUCTION}

The process of image digitization: creating a digital picture from a continuous image, involves two stages. One stage is image sampling, which determines the spatial resolution of a picture, and the second is image quantization, which determines the gray-level resolution at each pixel [6], [7]. Each of these stages had been treated separately, and multiresolution approaches in computer vision [8] as well as in signal processing [2] consider only spatial resolution.

A method introduced earlier to measure distances between histograms [10] is the basis to a unified treatment of spatial and graylevel resolution. This measure has also been found useful for reduction of gray-level resolution [3], and in this paper we present its applicability to the change of spatial resolution as well.

The ability to manipulate both spatial and gray-level resolutions can be very useful in several computer vision applications. Many py ramid algorithms, for example, use the effect that objects arbitrarily far away in the image eventually become local at one of the pyramid's levels. But spatial resampling usually has aliasing effects which introduce information not in the original image. These aliasing effects can be greatly reduced by increasing the color resolution while decreasing the spatial resolution. In other applications, if resolution is being reduced for compression purposes, it is beneficial to consider for a given compression rate the best combination of resolution reduction in both space and gray level.

In Section II, we introduce a graph-theoretical definition of the basic measure used. This will be followed by application to spatial resampling in Section III, and gray-level requantization in Section IV. Section V presents a hybrid treatment of space and gray-level resolutions.

Manuscript received April 13, 1987; revised February 13, 1989. This work was supported by a grant from the Israel Academy of Sciences.

The authors are with the Department of Computer Science. The Hebrew University of Jerusalem, 91904 Jerusalem, Israel.

IEEE Log Number 8928051.

\section{Distance Between Pictures}

A picture will be represented by a set of pixels in the plane. Each pixel can have a gray level in the range $[0, \cdots, N]$, and is represented by $N$ pebbles. When the gray-level of pixel $i$ is $g_{i}$ we assume that pixel $i$ has $g_{i}$ "white" pebbles and $N-g_{i}$ "black" pebbles. A picture is then regarded as a collection of pebbles, some white and some black, which are placed on the plane only at pixel locations, exactly $N$ pebbles per pixel.

A matching $\theta_{A B}$ is a one to one mapping between the pictures $A$ and $B$, i.e., each pebble of $A$ is matched to a pebble of $B$ having the same color. A matching is therefore defined only between pictures with same number of black (and white) pebbles, pictures with equal sums of gray level.

The cost of a matching $\theta_{A B}, \phi: \theta_{A B} \rightarrow{ }^{+}$, is a function from matchings to the nonnegative real numbers. Examples of reasonable cost functions for matchings are $\Sigma_{\left(a_{i}, b_{i}\right) \in \theta_{A B}}\left|a_{i}-b_{i}\right| ; \Sigma\left(a_{i}-\right.$ $\left.b_{i}\right)^{2} ; \operatorname{Max}\left\{\left|a_{i}-b_{i}\right|\right\}$ where $\left|a_{i}-b_{i}\right|$ is the distance between the locations of pebbles $a_{i}$ and $b_{i}$. A matching $\theta_{A B}$ can be regarded as an algorithm to change picture $A$ into picture $B$ : for each $\left(a_{i}, b_{i}\right) \in$ $\theta_{A B}$, move pebble $a_{i} \in A$ to the location of $b_{i}$. The cost of this single pebble move is $\left|a_{i}-b_{i}\right|$. The cost of the matching can be regarded as the cost of the entire transformation of $A$ and $B$, and is computed from the costs of individual movements in one of the three ways described earlier (sum, sum of squares, or max).

Let $\theta$ be the set of all matchings between pictures $A$ and $B$, and let $\theta \in \Theta$ be of minimal cost. This minimal cost matching will be called the transformation of $A$ and $B$, and the $\operatorname{cost} \phi\left(\theta_{A B}\right)$ will be called the match-distance between $A$ and $B$ and denoted by $\rho(A$, $B$ ). The transformation is not always unique, and in such cases can be chosen arbitrarily from all minimal cost candidates. The match distance is unique with respect to the cost function $\phi$.

When two pictures have same number of pixels as well as pebbles, it is enough to consider the white pebbles only. Let $\left(a_{i}, b_{i}\right)$ be a pair of white pebbles in the minimal matching, designating a move of one white pebble from $a_{i}$ to $B_{i}$. As each pixel has $N$ pcbbles, there is a minimal matching with a black pebble from the location of $b_{i}$ moved back to the location of $a_{i} .\left(a_{i}, b_{i}\right) \in \theta_{A B}$ can therefore be regarded as an exchange of a white pebble from the location of $a_{i}$ and a black pebble from the location of $b_{i}$, leaving the total number of pebbles at each pixel unchanged.

Example: We will compute the distance $\rho=\Sigma\left|a_{i}-b_{i}\right|$ for the following three pictures:

$$
f_{1}=\left[\begin{array}{lll}
0 & 1 & 0 \\
0 & 1 & 0 \\
0 & 1 & 0
\end{array}\right] ; \quad f_{2}=\left[\begin{array}{lll}
1 & 0 & 0 \\
0 & 1 & 0 \\
0 & 0 & 1
\end{array}\right] ; \quad f_{3}=\left[\begin{array}{lll}
0 & 0 & 0 \\
1 & 1 & 1 \\
0 & 0 & 0
\end{array}\right] \text {. }
$$

The numbers at the above example represent the gray level, or the number of white pebbles, at a pixel.

The minimal matchings of white pebbles are

$$
\begin{aligned}
& \theta_{f_{1}, f}=\{\langle(1,0),(0,0)\rangle,\langle(1,1),(1,1)\rangle,\langle(1,2),(2,2)\rangle\} \\
& \theta_{f, j}=\{\langle(0,0),(0,1)\rangle,\langle(1,1),(1,1)\rangle,\langle(2,2),(2,1)\rangle\} \\
& \theta_{f_{1}, f}=\{\langle(1,0),(0,1)\rangle,\langle(1,1),(1,1)\rangle,\langle(1,2),(2,1)\rangle\}
\end{aligned}
$$

$\theta_{f_{1}, f}$ specifies that in order to change $f_{1}$ into $f_{2}$ the white pebble at location $(1,0)$ is exchanged with the black pebble at location $(0$, $0)$, nothing happens at the center location $(1,1)$, and the white pebble at location $(1,2)$ is exchanged with the black pebble at 
location $(2,2)$. The cost of each exchange is 2 (moving two pebbles at distance of 1 each), yielding the total cost of $\rho\left(f_{2}, f_{3}\right)=4$ and $\rho\left(f_{1}, f_{3}\right)=4 \sqrt{2}$. Using regular vector distance all three pictures are equally far apart, but using the match-distance yields larger distance between $f_{1}$ and $f_{3}$. This result is in better agreement with our intuitive notion of distance between pictures.

\section{SPATIAL RESAMPLing}

In spatial resampling, given a set $X=\left\{x_{i}\right\}$ of original pixels, and another set of pixels $Y=\left\{y_{i}\right\}$ (the target picture), distribute the gray levels ("pebbles") from the pixels of $X$ to the pixels of $Y$, so that the picture $Y$ is as similar as possible to the picture $X$. We move white as well as black pebbles, and as $Y$ should be a picture too, each of its pixels receives equal number of pebbles.

As in all resampling methods, we assume that the gray level at a pixel $y_{j}$ is a linear combination of the gray levels of pixels $x_{i}$, with weights $w_{i j}$

$$
G\left(y_{j}\right)=\sum_{i} w_{l j} G\left(x_{i}\right)
$$

The cost of this resampling transformation is computed as follows. As each pixel $x_{i}$ has $N$ pebbles, the cost of contributing $w_{i j}$ of them to $y_{j}$ is $N \cdot w_{i j} \cdot d_{i j}$ where $d_{i j}$ is the distance between $x_{i}$ and $y_{j}$. The total cost of the resampling transformation, under the sum cost, is

$$
N \cdot \sum_{i} \cdot \sum_{j} w_{i j} \cdot d_{i j}
$$

We wish to minimize the above cost, while maintaining the following conditions:

1) The pixels in $X$ contribute all their pebbles, i.e.,

$$
\sum_{j} w_{i j}=1 \text {. }
$$

2) All pixels of $Y$ receive equal number of pebbles, $|X| \cdot N /|Y|$, i.e.,

$$
\sum_{i} w_{i j}=\frac{|X|}{|Y|}
$$

where $|X|$ is the number of pixels in the picture $X$.

3) The gray level of pixel $y_{j}$ depends more on pixels $x_{i}$ that are closer to it than on further ones.

We will find the coefficients of the transformation by using linear programming. As we move complete pebbles, we need the coefficients $w_{i j}$ to be integers. We can get this by normalizing the coefficients and rewriting the linear program with coefficients $r_{i j}$ as follows:

$$
\begin{aligned}
& \text { Minimize the cost } \sum_{i} \sum_{j} r_{i j} \cdot d_{i j} \\
& \text { such that for each } x_{i} \quad \sum_{j} r_{i j}=|Y| \\
& \text { and for each } y_{j} \quad \sum_{i} r_{i j}=|X| .
\end{aligned}
$$

Solving the above linear program for the $r_{i j}$ 's, we can compute the white pebbles at $Y$ (the gray levels) by $G\left(y_{j}\right)=\Sigma_{i} r_{i j} \cdot G\left(x_{i}\right)$. $G\left(y_{j}\right)$ is now in the range $[0, \cdots, N,|X|]$. If we wish to normalize the gray levels of $Y$ to be in the range of $[0, \cdots, N]$ as in $X$, we can normalize $G\left(y_{j}\right)$

$$
G^{\prime}\left(y_{j}\right)=\frac{\sum r_{i j} \cdot G\left(x_{i}\right)}{|X|} .
$$

In Section $\mathrm{V}$, we will discuss the case where we wish to change the gray-level range of the target image as well as its size.

For the pairwise distance $d_{i j}$ between points $x_{i}$ and $y_{j}$ we can use different measures, like the city-block, chess-board, or Euclidean distances.

A property of this linear program is that there is always a min-

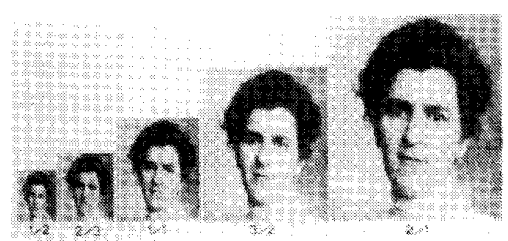

Fig. 1. Examples for spatial resampling. The original image, size $128 \times$ 128 , is at the center. Other images arc with sides of $1 / 2,2 / 3,3 / 2$, and 2 times the side of the original picture.

imum in which all the $r_{i j}$ are integer. This follows from the fact that the matrix of the equalities is unimodular, as it describes a bipartite graph between the pebbles of $X$ and $Y$ [4].

An example of this method for the expansion and contraction of pictures is shown in Fig. 1. Although in Fig. 1 all images are on a rectangular grid, the change of scale is arbitrary, and is not restricted by integral multiplications. The method is also not restricted to regular grids, and is defined on any two sets of pixels. If can transform a square grid, for example, into an hexagonal grid, $\log$ polar, etc.

This resampling method can also accommodate varying weights on pixels, similarly to the method described in [5]. This approach gives higher weight to pixels on high information regions, like edges or other busy regions. A morc claborate treatment of weighted pixels can be found in [11].

\section{Gray-Level Requantization}

Reduction of the gray-level range of an image such that the resulting picture will be as similar as possible to the original picture is considered. We use the match-distance to measure the quality of reduction of gray-level resolution, enabling the comparison of graylevel resolution to spatial resolution as will be done in the next chapter.

This requantization method, first described in [3], can take an image in any gray-level range and transform it optimally to another image of the same size in any desired gray-level range. The benefit of this process is the cost it associates for the requantization. Such cost is unavailable with other requantization methods. The computational cost of arbitrary gray-level changes is high. More computationally efficient is the subproblem of the reduction of the graylevel range from $[0, \cdots, 2 N]$ to $[0, \cdots, N]$. Repetition of this process will result in a binary halftone.

Given a picture with gray-level range of $[0, \cdots, 2 N]$, reducing the gray-level range to $[0, \cdots, N]$ is equivalent to leaving the range $[0, \cdots, 2 N]$, but having only even gray levels, i.e., $[0,2,4, \cdots, 2 N]$. Changing from range of $[0,2,4, \cdots, 2 N]$ to $[0, \cdots, N]$ is accomplished easily using a division by two. In order to change all gray levels to be even, only pixels with odd gray levels are considered. We pair together such pixels, and they exchange within each pair a white pebble for a black pebble. After such exchange, both pixels in the pair get an even gray level. We should remember that the gray level is also the number of white pebbles at a pixel.

The pairing of pixels with odd gray levels is done minimizing its cost: minimize the sum, over all pairs, of pairwise distances. As pairwise distances we consider the distance between the two pixels of the pair where the distance can be Euclidean, chessboard. city block, etc.

The algorithm for reducing the gray-level range from $[0, \cdots$, $2 N]$ to $[0, \cdots, N]$ is summarized as follows.

1) Optimally pair pixels with odd gray level. Algorithms for such pairing will be discussed later.

2) In all pairs, exchange one white pebble for a black pebble. The cost of such pairwise exchange is twice the distance between the pixels in the pair. After this step all gray levels are cven.

3) Divide the gray levels by two. 
The following example can help to clarify the algorithm. Given the original picture:

$$
\left[\begin{array}{lllll}
7 & 5 & 4 & 3 & 1 \\
7 & 5 & 4 & 3 & 1 \\
7 & 5 & 4 & 3 & 1 \\
7 & 5 & 4 & 3 & 1 \\
7 & 5 & 4 & 3 & 1
\end{array}\right]
$$

We pair the pixels with odd gray levels

$$
\left[\begin{array}{ccccc}
7 & 5 & 4 & 3-1 \\
1 & 1 & & \\
7 & 5 & 4 & 3-1 \\
7 & -5 & 4 & 3 & 1 \\
7 & 5 & 4 & 3 & 1 \\
1 & 1 & & 1 \\
7 & 5 & 4 & 3-1
\end{array}\right] .
$$

Within each pair we exchange a white for black pebble

$$
\left[\begin{array}{ccccc}
6 & 6 & 4 & 2-2 \\
1 & 1 & & \\
8 & 4 & 4 & 4-0 \\
6 & -6 & 4 & 2 & 2 \\
8 & 4 & 4 & 4 & 0 \\
1 & 1 & & \\
6 & 6 & 4 & 2-2
\end{array}\right] .
$$

We now divide the resulting gray level by two to get the final requantization

$$
\left[\begin{array}{lllll}
3 & 3 & 2 & 1 & 1 \\
4 & 2 & 2 & 2 & 0 \\
3 & 3 & 2 & 1 & 1 \\
4 & 2 & 2 & 2 & 0 \\
3 & 3 & 2 & 1 & 1
\end{array}\right]
$$

Step 1, which computes the minimal cost pairing, is the step with highest computational complexity. Good approximations to the minimal cost pairing can be found by heuristic methods, with linear time complexity [1], [9]. Exact proof that this pairing algorithm finds the closest picture in terms of the minimal match-distance can be found in [3]. Example of the gray-level requantization is shown in Fig. 2. The process is an iterative process, where at each iteration the upper bound of the gray-level range is reduced by half.

\section{Hybrid Spatial and Gray-Level Resolution}

The tool of match-distance enables us to perform and compare different combinations of reduction of resolution. We regard the total resolution of a picture as the number of bits used for its matrix representation. As an example, assume we wish to reduce by four the total resolution devoted to a picture of size 64 by 64 with 8 bits per pixel, denoted by $(64 \times 64 \times 8)$. Some of the possibilities include pure spatial resolution reduction: $(32 \times 32 \times 8)$; pure gray-level resolution reduction: $(64 \times 64 \times 2)$; and two hybrid combinations: $(37 \times 37 \times 6)$ and $(45 \times 45 \times 4)$. The hybrid combinations do not give an exact reduction by four, but come very close. Given a picture and a desired total resolution reduction, the match distance can give us a tool to select among the different options.

The hybrid resolution reduction can be formulated as follows. Given a set $X=\left\{x_{i}\right\}$ of original pixels, $N$ pebbles in each pixel, and a target picture $Y=\left\{y_{i}\right\}$ with $K$ pebbles in each pixel, find the picture $Y$ that will most resemble $X$. (a)

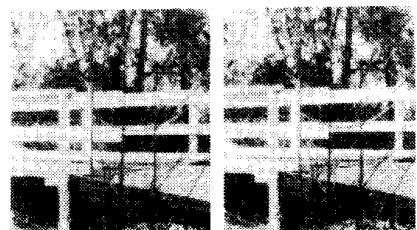

(b)

(c)

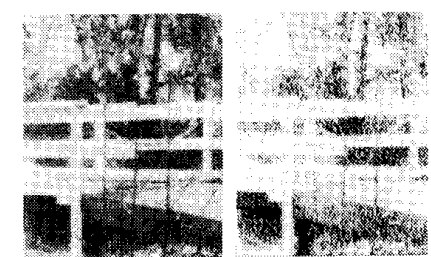

Fig. 2. Comparing the original image with gray-level range [0. 256] (a) with the last three iterations of the requantization algorithm: gray-level range $[0.4](b)$, gray-level range $[0.2](\mathrm{c})$, and gray-level range $[0 . .1](\mathrm{d})$. (b)

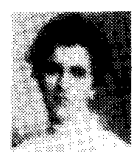

(d)

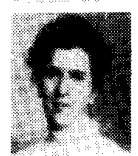

(a)
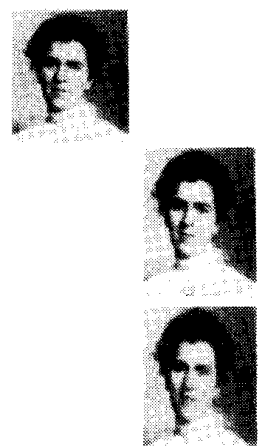

Fig. 3. Different combinations of same total resolution reduction. All images were spatially enlarged for display purposes to the size of the original picture. All reduced images need about the same number of bits for representation. (a) Original picture, size $144 \times 144$, gray-level range [0. 256]. (b) Pure spatial resolution reduction, size $48 \times 48$, graylevel range $[0 . .256]$. (c) size $84 \times 84$, range $[0.4]$. (d) size $72 \times$ 72 , range $[0.8]$. (e) size $60 \times 60$, range $[0.16]$.

The number of pebbles in $X$ is $|X| \cdot N$, and the number of pebbles in $Y$ is $|Y| \cdot K$. As the number of pebbles in both pictures is different, $|X| \cdot N \neq|Y| \cdot K$, we use their least common multiple, and find the smallest $\alpha, \beta$, such that $|X| \cdot N \cdot \alpha=|Y| \cdot K \cdot \beta$. We multiply the number of pebbles in each pixel of $X$ by $\alpha$ (and have $N \cdot \alpha$ pebbles per pixel ), and the number of pebbles in each pixels of $Y$ by $\beta$ (to have $K \cdot \beta$ pebbles per pixel). We then map (a minimal cost mapping) the pebbles of $X$ and $Y$. We perform this mapping under the constraint that pixels in $Y$ get only $k \cdot \beta,(k=$ $0,1, \cdots, K)$ white pebbles. With this constraint, simple division of the number of pebbles in $Y$ by $\beta$ will result in the desired range of $K$ pebbles per pixel. Without this constraint the division would rcsult in fractions of white and black pebbles.

The complexity of the algorithm to solve the mapping described above is very high, and at this moment we do not have an efficient algorithm to perform it completely. To get approximated results we use two stages. In the first stage, we change the spatial resolution using the linear program of Section III, and get $N \cdot|X|$ pebbles at each pixel of $Y$. In the second stage, we use the halftoning technique of Section IV to reduce the number of pebbles to the desired $K$. Actually, if $K \geq N$, simple division is satisfactory. Only when $K<N$ we have to use the halftoning technique.

Fig. 3-4 show combined application of space and gray level resolutions. Fig. 3 displays a portrait, while Fig. 4 is a texture picture. 


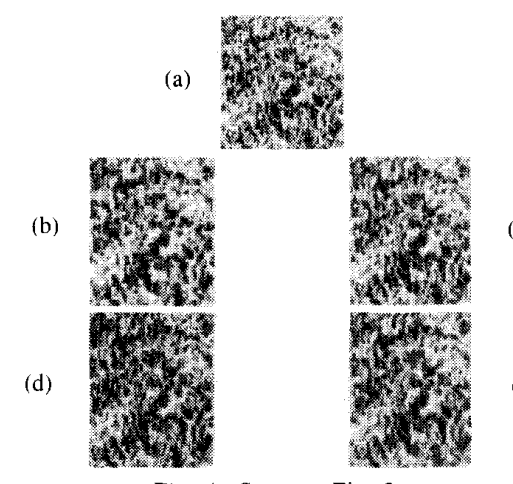

Fig. 4. Same as Fig. 3.

\section{Concluding Remarks}

This paper presents a hybrid treatment of resolution, and the possibilities in trading spatial for gray-level resolution and vice versa. The theoretical foundation of the match-distance is strong, but it is computationally very expensive. As future directions, we see either the simplification of the match-distance to a measure more easily computed, or finding efficient and accurate estimates for the match-distance. We have no doubt, however, that this approach to the treatment of resolution will prove useful to the future generation of multiresolution methods.

\section{REFERENCES}

[1] M. Ben-Or. "Lower bounds for algebraic computation trees," in Proc. 15th ACM Symp. Theory Comput., Boston, MA, 1983, pp. 8086.

[2] R. E. Crochiere and L. R. Rabiner, Multirate Digital Signal Processing. Englewood Cliffs. NJ: Prentice-Hall. 1983.

[3] M. Werman and S. Peleg, "Halftoning as optimal quantization," in Proc. Eighth Int. Conf. Pattern Recog., Paris, France, Oct. 1986, pp. 1114-1116.

[4] C. K. Papadimitriou and K. Steiglitz. Combinatorial Optimization: Algorithms and Complexity. Englewood, Cliffs, NJ: Prentice-Hall, 1982.

[5] S. Peleg, O. Federbush, and R. A. Hummel. "Custom-Made Pyramids." in Parallel Computer Vision. New York: Academic, 1987. pp. 125-146.

[6] W. K. Pratt, Digital Image Processing. New York: Wiley-Interscicnce, 1978.

[7] A. Rosenfeld and A. C. Kak, Digital Picture Processing (2nd ed.). New York: Academic. 1982

[8] A. Rosenteld. Multiresolution Image Processing and Applications. New York: Springer Verlag. 1984.

[9] K. J. Supowit and E. M. Reingold, "Divide and conquer heuristics for minimum weighted matching." vol. 12, 1983.

[10] M. Werman. S. Peleg, and A. Rosenfeld, "A distance metric for multidimensional histograms," Comput. Vision, Graph., Image Processing, vol. 32, pp. 328-336, Dec. 1985.

[11] M. Werman, "A distance measure for the comparison of pictures and shapes." Ph.D. dissertation. The Hebrew Univ. Jerusalem, 1986

\section{Quadtree-Structured Linear Prediction Models for Image Sequence Processing}

\section{PETER STROBACH}

Abstract-This correspondence summarizes a study on two-dimensional linear prediction models for image sequence processing and its

Manuscript reccived Fcbruary 23. 1988; revised January 20, 1989. Recommended for acceptance by A. K. Jain.

The author is with SIEMENS AG, Zentralbereich Forschung und Technik. D-8000 Munchen 83, West Germany.

IEEE Log Number 8928045 application to change detection and scene coding. The study was focused on two major areas: Two-dimensional joint process modeling of interframe relationships, the derivation of computationally efficient matching algorithms and the implementation of a block-adaptive interframe predictor for use in interframe predictive coding and change detection. In the presented approach, the spatial nonstationarity is handled by an underlying quadtree segmentation structure. A maximum likelihood criterion and a simpler minimum variance criterion are discussed as detection and segmentation rules.

The results of this research indicate that a constrained joint process model involving only a single gain-parameter and a shift parameter is the best tradeoff between performance and computational complexity.

Index Terms-Change detection, image segmentation, image sequence processing, quadtrees.

\section{INTRODUCTION}

Image sequences capture not only static, but also dynamic aspects of a recorded scene. The evaluation or coding of image sequences therefore requires algorithms for extraction and interpretation of dynamic relations between successive frames in an image sequence, such as velocity and shape of moving brightness patterns, as well as, changes in illumination.

Algorithms facilitating the extraction of the above mentioned features from the pixel information can be split in two basic categories. The first class of algorithms is based on the iterative computation and evaluation of optical flow fields [1]-[6] which describe the distribution of apparent velocities of movement of brightness patterns in an image. Consequently, discontinuities in the optical How can help segmenting images into regions that correspond to different objects [2], [3], [5]. In the optical flow concept, an inherent difficulty arises when the illumination changes with time. In this case, the continuity equation of optical flow [1] is violated which can cause serious errors in the estimated optical flow (displacement vector) field.

Therefore, a second class of scene modeling algorithms which derives from the idea of correlating matching [7] has gained considerable importance. Recall that various types of different block matching (BM) algorithms [7]-[9] have been applied with great success in the domain of image sequence coding. The closer analysis presented in this paper reveals that a larger number of recently proposed BM algorithms appear as special cases of a more general image sequence model in which the relationship between two successive frames in a sequence is modeled by a two-dimensional block adaptive least-squares joint process filter [10]-[12]. This approach can also be brought in relation with some previous work of other authors where linear prediction models were applied with great success on the problem of intraframe (in-field) image coding [13], [14]. As an important novelty, we present the embedding of the described linear prediction models in an adaptive quadtree segmentation structure [15]-[17] which helps to handle spatial nonstationarities. In this concept, two segmentation rules are discussed. This is, first, a simple minimum variance detector and, second, we have extended the well-known generalized likelihood ratio (GLR) detector of Willsky and Jones [18] to the problem of change detection and spatial segmentation.

The correspondence is organized as follows. In Section II, we provide the necessary definitions and properties to aid in the understanding of the subsequent sections. A constrained joint process model is used for modeling of the relationships between subsequent images in a scene. In Section III, we present several approaches on the important problem of segmenting the interframe information into stationary regions of uniform size and shape utilizing a quad tree segmentation structure. Section IV discusses two applications of the theoretical results obtained in the earlier sections.

\section{The Constrained Joint Process Model}

This section reviews the basic concept of joint process estimation which is a well established linear prediction technique for de- 